\title{
Acessibilidade Comunicacional em um Objeto de Aprendizagem Matemática para Estudantes Surdos
}

\author{
Amanda Maria Domingos de Oliveira ${ }^{1}$, Eli Sales Muniz Lima ${ }^{2}$, Flávia Roldan \\ Viana $^{1}$, Gabriel Vieira Barreto ${ }^{1}$, Jason Willyan Castro do Nascimento ${ }^{2}$, Moisés \\ Oliveira Catonio de Araújo ${ }^{2}$
}

${ }^{1}$ Centro de Educação - Universidade Federal do Rio Grande do Norte (UFRN) Av. Sen. Salgado Filho, 3000 - Lagoa Nova - CEP 59.078-970 - Natal - RN - Brazil

${ }^{2}$ Instituto Metrópole Digital - Universidade Federal do Rio Grande do Norte (UFRN) Av. Sen. Salgado Filho, 3000 - Lagoa Nova - CEP 59.078-970 - Natal - RN - Brazil

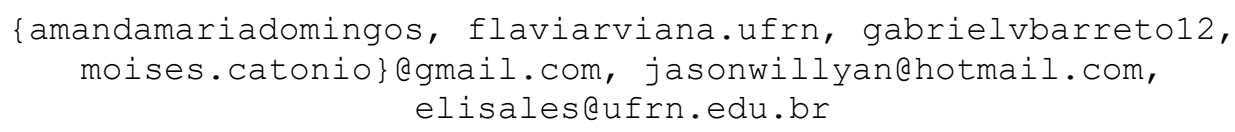

\begin{abstract}
Aware of the need to provide deaf students with access to information and educational technological resources that may assist in their teaching and learning process, this study aims to discuss the processes of implementing communicational accessibility in the Learning Object (OA) Conta Kg The development process took place through the collaborative work of an interdisciplinary group that brings together students of Pedagogy and Information Technology. Accessibility was achieved through the inclusion of videos in Libras for all texts present in the OA, using the Unity $3 D$ game engine.

Resumo. Cientes da necessidade de proporcionar ao aluno surdo o acesso às informações e a recursos tecnológicos educativos que venham a auxiliar no seu processo de ensino e aprendizagem, este estudo tem o objetivo de discorrer sobre os processos de implementação da acessibilidade comunicacional no Objeto de Aprendizagem (OA) Conta Kg. O processo de desenvolvimento se deu através do trabalho colaborativo de um grupo interdisciplinar que reúne estudantes de Pedagogia e Tecnologia da Informação. A acessibilidade foi feita por meio da inclusão de vídeos em Libras para todos os textos presentes no OA, utilizando-se o motor de jogos Unity $3 D$.
\end{abstract}

\section{Introdução}

A surdez é definida pela redução ou ausência da capacidade de percepção dos sons. Segundo Gagliardi e Barella (1986) a surdez pode ser entendida como uma privação sensorial, cujo sintoma comum é a não reação diante de um estímulo sonoro, caracterizada pela deficiência auditiva, que pode ser expressa por uma ausência total ou parcial da audição. De acordo com a Organização Mundial de Saúde (OMS) cerca de 460 milhões de pessoas no mundo tem perda auditiva incapacitante, das quais 34 milhões são crianças [Who 2020].

Ao ter sua capacidade de percepção sonora reduzida, a pessoa surda passa a compreender e interagir com o mundo através de suas experiências visuais e a se comunicar por meio de uma língua gestual-visual, a Língua de Sinais (LS). No Brasil, foi 
estabelecido por lei, desde 2002, a Língua Brasileira de Sinais (Libras ${ }^{1}$ ) como meio legal de comunicação associado a comunidade surda do Brasil.

No caso do aprendizado escolar, a dificuldade de aprendizagem da Matemática também acomete os estudantes surdos. Contudo, a surdez não é causa direta para essas dificuldades e, sim, fator de risco. Dessa forma, é possível afirmar que existem experiências ligadas à surdez que podem dificultar a aprendizagem matemática [Viana e Barreto 2014]. Estudos [Viana e Barreto 2014; Coutinho 2015; Viana et al 2018] têm demonstrado que os estudantes surdos têm desempenho mais baixos que os estudantes ouvintes. Porém, essa forma de perceber o mundo e acessar as informações e conhecimentos não interfere na capacidade cognitiva de indivíduos surdos.

A pesquisa de Obilade (2015) que trata de uma comparação entre a capacidade cognitiva de surdos e ouvintes, a partir de uma pesquisa realizada com estudantes de escolas primárias na Nigéria, reforça tal afirmação. O pesquisador utilizou um teste de inteligência padronizado sem referências verbais. Após a etapa de testes os dados foram utilizados para confrontar as variáveis que pudessem interferir no resultado do estudo, e, conforme apresentado pela pesquisa, não há diferenças significativas com relação às funções cognitivas de surdos e ouvintes.

Diante disso, pode-se notar que para pessoas com surdez há uma necessidade da inclusão educacional para que este tenha acesso à educação de maneira efetiva, como é promulgado no Art. 4 da Lei ${ }^{\circ} 10.436$ :

\begin{abstract}
Art. $4^{\circ} \mathrm{O}$ sistema educacional federal e os sistemas educacionais estaduais, municipais e do Distrito Federal devem garantir a inclusão nos cursos de formação de Educação Especial, de Fonoaudiologia e de Magistério, em seus níveis médio e superior, do ensino da Língua Brasileira de Sinais - Libras, como parte integrante dos Parâmetros Curriculares Nacionais - PCNs, conforme legislação vigente.
\end{abstract}

No atual contexto inclusivo é preciso garantir a igualdade e a equidade na aprendizagem de pessoas surdas mas, infelizmente, ainda são encontradas barreiras pela falta da disponibilidade de recursos pedagógicos acessíveis que garantam a este público o acesso às informações e ao conhecimento, que, muitas vezes, é excludente à pessoas com deficiência. A exemplo, nos ambientes virtuais de aprendizagem (AVAs) é evidente a falta de acessibilidade para pessoas surdas ou ensurdecidas, como por exemplo: a adequação a textos curtos; a introdução de imagens para iniciar um conceito; a tradução em língua de sinais, no caso do Brasil, a Libras; textos alternativos e navegação acessível.

Com o surgimento e popularização dos AVAs e as regulamentações políticas e sociais para a inclusão e acessibilidade, iniciam-se estudos para tornar a educação virtual disponível a todos os públicos. Neste contexto, as novas tecnologias surgem como importantes aliados no processo de aprendizagem dos surdos, uma vez que através de recursos tecnológicos é possível adequar esses ambientes a qualquer modo de comunicação, levando em conta a particularidade de cada pessoa. A exemplo, as Diretrizes de Acessibilidade de Conteúdos $W e b$ em suas duas versões 1.0 e 2.0 destacam a importância de que para uma integral acessibilidade na internet é fundamental

\footnotetext{
${ }^{1}$ Adota-se a norma da Língua Portuguesa, segundo a qual se uma sigla for pronunciável como se fosse uma palavra ela deve ser escrita com apenas a inicial maiúscula; e se ela não for pronunciável como uma palavra, mas apenas como uma série de letras deve ser escrita em maiúsculas.
} 
acrescentar as técnicas de acessibilidade associadas ao Web Content Accessibility Guidelines - WCAG e suas recomendações.

Tais recomendações foram publicadas pela Web Accessibility Initiative do World Web Consortium (W3C). Entretanto, Flor, Vanzin, Ulbricht [2013, p. 162] atentam para o fato de que:

\footnotetext{
“[...] as prescrições contidas nestas diretrizes quanto ao acesso de surdos possuem o enfoque das pessoas sem deficiência, que não dão a devida relevância da Língua de Sinais para a acessibilidade na web, predominando as recomendações para legendas textuais".
}

Porém, no Brasil, a partir da Lei $\mathrm{n}^{\mathrm{o}} 10.436$ de 24 de abril de 2002, pessoas surdas passam a ser reconhecidas como possuidoras de uma diferença linguística, que utilizam a Libras como meio principal de comunicação, sendo esta sua primeira língua, sua língua materna. Pereira (2014) afirma que muitos surdos não possuem o domínio da Língua Portuguesa, apresentando muitas vezes o desinteresse pela leitura devido às estratégias tradicionais de ensino que conduzem os surdos a decodificar algumas palavras, mas sem terem o domínio da compreensão textual.

É preciso considerar que a pessoa surda é um sujeito cognoscente de aprendizagem, essencialmente, visual, tendo em vista que

\section{[...] tudo que ele aprende é a partir do que vê. Precisamos, contudo, ajudá-lo a amplificar sua análise dessas imagens a decodificá-las, a fim de que ele possa enriquecer sua seleção e escolhas dos "textos" que vai ler, ampliando seu vocabulário e instrumentalizando-o a pensar e agir com maior autonomia. [Domingues 2006, p. 23].}

Entretanto, nos ambientes da web, a Libras não é a língua mais difundida. Para que o sujeito surdo, usuário da língua de sinais como primeira língua, tenha acesso às informações, a presença desta língua é essencial. De acordo com Góes \& Gomes (2011). os recursos de acessibilidade existentes para usuários surdos na rede mundial de computadores, são, em sua maioria, videos com legendas explicativas em português - $\mathrm{o}$ que não contempla a realidade da parcela maior deste grupo social.

Pesquisas na área da acessibilidade e inclusão [Bahia e Trindade 2010; Xavier 2011; Giroto, Poker e Omote 2012; Rodrigues, 2012; Santarosa e Conforto, 2015; Viana et al, 2019], indicam que as tecnologias educativas auxiliam na promoção da inclusão, propiciando facilidades que tornam a aprendizagem mais dinâmica e interessante.

Nesse contexto, em 2019, o grupo de pesquisa OBAMA, que tem como função apresentar a alunos e professores possibilidades pedagógicas com a utilização de Objetos de Aprendizagem (OA), a fim de utilizar as Tecnologias Digitais da Informação e Comunicação (TDIC) para auxílio da Educação Matemática [Maia et al, 2017] criou um GT (Grupo de Trabalho) de acessibilidade, denominado OBAMA - CARE (Componente de Acessibilidade e Responsividade), para desenvolver ações que promovam as práticas inclusivas digitais. Faz parte dessas ações o desenvolvimento de OAs acessíveis a estudantes surdos que promovam, entre outras aprendizagens, o letramento estatístico, e, assim, promova a inclusão da Educação Matemática. A proposta de desenvolvimento dos OA, está no contexto das ações da equipe que desenvolveu a Plataforma OBAMA. Entretanto,

"[...] ainda, precisam estar muito claras as metas a serem alcançadas com o uso desses recursos. Isto requer a necessidade de que se compreenda efetivamente 
os princípios e propostas implicadas na educação inclusiva, construindo atitudes genuinamente acolhedoras das diferenças e favoráveis à inclusão". [Giroto, Poker e Omote, 2012, p. 22].

Partimos da hipótese que as TDIC, pelas inesgotáveis possibilidades de construção de recursos, podem contribuir para uma aprendizagem inclusiva, por possuírem diversificadas aplicabilidades no âmbito educacional. Para isso, é preciso sensibilizar os profissionais que atuam nessa área para que suas ações didáticopedagógicas sejam orientadas pelas potencialidades de seus estudantes e não pelas suas limitações.

Entretanto, para que um Objeto de Aprendizagem possa atender a seu papel de apoiar a aprendizagem, faz-se necessário que seja acessível a todos os seus usuários. Isso implica, segundo Melo (2015, p. 94-95), “[...] flexibilidade para acomodar, na maior extensão possível, a multiplicidade das diferenças entre os aprendizes". A pesquisadora ainda sugere que, para a promoção da acessibilidade é estratégico que o OA possua aderência ao Desenho Universal, compatibilidade com recursos de Tecnologia Assistiva e esteja de acordo com os quatro princípios de acessibilidade web, amplamente difundidos pelo W3C - World Wide Web Consortium (2008). A autora esclarece que

“[...] se um OA do tipo imagem não apresenta texto alternativo, uma pessoa
cega não perceberá a informação veiculada, ferindo princípios do Desenho
Universal como 'Uso equitativo' e 'Informação perceptível'. Se um OA do tipo
simulação, hipertexto ou software não oferecer alternativas para sua operação,
uma pessoa com mobilidade reduzida poderá ter dificuldade de manipulá-la
com suas tecnologias de acesso, ferindo princípios do Desenho Universal como
'Uso equitativo' e 'Flexibilidade no Uso'. Se um OA do tipo áudio, vídeo ou
animação não possuir transcrição em texto ou alternativa em Libras para o
conteúdo sonoro, oferecerá barreiras à percepção e à compreensão por pessoas
com deficiência auditiva e por pessoas surdas, ferindo princípios do Desenho
Universal como 'Uso equitativo', 'Informação perceptível' e 'Uso simples e
intuitivo'. [Melo, 2015, p. 98, GRIFOS DA AUTORA].

Após a criação do GT dedicado à acessibilidade, foi iniciado um ciclo de ações para os membros, uma dessas ações era a criação de um OA para matemática que fosse acessível a pessoas com deficiência. Por ser um espectro amplo de especificidades, optamos por focar na acessibilidade comunicacional para pessoas surdas. Nesse sentido, uma das primeiras ações do GT OBAMA-CARE foi inserir recursos de acessibilidade a um OA existe. Foi selecionado, então, um OA cujo (i) sua utilização já houvesse sido validada, (ii) possuísse licença livre para que pudesse ser adaptado, reutilizado e redistribuído, e (iii) e fosse familiar entre os membros do grupo, referente a sua utilização em sala de aula. Dessa forma, o Conta $\mathrm{Kg}^{2}$ foi o OA selecionado. As atividades propostas no OA Conta Kg contemplam o desenvolvimento das habilidades previstas em Unidades Temáticas como Probabilidade e Estatística e Números, segundo a Base Nacional Comum Curricular (BNCC) [Brasil, 2017].

Assim, este trabalho objetiva discorrer sobre os processos de implementação da acessibilidade comunicacional no Objeto de Aprendizagem (OA) Conta Kg, para favorecer ao usuário surdo o acesso às informações e possibilitar o conhecimento

\footnotetext{
${ }^{2}$ A versão selecionada para inclusão de acessibilidade pode ser acessada em: $<$ https://softwareducativo.github.io/Contakg/>
} 
IX Congresso Brasileiro de Informática na Educação (CBIE 2020)

Anais do XXVI Workshop de Informática na Escola (WIE 2020)

matemático, podendo vir, assim, a auxiliar o processo de ensino e aprendizagem desses estudantes.

\section{Processo de Implementação da Acessibilidade Comunicacional}

O aporte metodológico do processo de implementação da acessibilidade comunicacional no OA repousa no paradigma epistemológico para a condução de pesquisas tecnológicas, o Design Science que orienta o método de pesquisa Design Science Research (DSR). De acordo com Venable (2006); Myers e Venable (2014); Dresch, Lacerda e Antunes-Junior (2015), o método de pesquisa DSR é encaminhado à resolução de problemas, teóricos ou práticos, no qual busca a partir do entendimento de uma situação, gerar conhecimento aplicável para a solução do problema identificado, construir/criar e avaliar artefatos que permitam transformar a realidade, alterando suas condições para resultados viáveis e melhores.

\subsection{Etapas e atividades do processo de implementação}

A seguir informaremos sobre as etapas para a implementação da acessibilidade comunicacional para surdos no Objeto de Aprendizagem (OA) Conta Kg, relatando as dificuldades e soluções de acessibilidade encontradas.

Considerando que as TDICs somam esforços no campo da acessibilidade, elegemos, para efeito de nossos estudos, três esferas de atuação epistemológicas: i) A esfera das tecnologias educacionais, tendo como referência as pesquisas e os trabalhos desenvolvidos pela equipe OBAMA, com ênfase na acessibilidade da Plataforma OBAMA e na produção de materiais didáticos acessíveis para apoio às atividades pedagógicas, levando em conta as tecnologias assistivas e as especificidades linguísticas do sujeito surdo; ii) A esfera da acessibilidade, considerando as pretensões inclusivas do GT OBAMA - CARE quanto a acessibilidade da plataforma, o desenvolvimento de objetos de aprendizagem, elaboração de material didático, formações docentes, plano de aula inclusivo, entre outras atividades; e iii) A esfera da inclusão que está relacionada a outros projetos do GT OBAMA - CARE realizados em ambientes educacionais escolares e não escolares que mobilizam práticas e processos na área da educação de surdos.

Um dos primeiros obstáculos encontrados no desenvolvimento deste trabalho foi o não domínio da tecnologia em que foi desenvolvida a primeira versão do Conta $\mathrm{Kg}$, por parte dos integrantes da equipe GT OBAMA - CARE. A partir disso, foi realizada uma nova implementação do OA. Para isso escolhemos a plataforma Unity $3 \mathrm{D}$, um motor de jogos que possibilita a exportação do produto final para diversas plataformas, seja mobile, Web ou Desktop. Plataformas como essa aparecem como um poderoso pacote de software que permite aos desenvolvedores criar seus jogos e aplicativos usando módulos preexistentes de renderização 2D / 3D, física de jogos, manipulação de entradas, entre outras facilidades [Lewis e Jacobson 2002].

Após a definição da tecnologia que seria utilizada, foi realizada uma análise da literatura e de OA acessíveis com vistas a identificar os principais elementos de acessibilidade na web para usuários surdos. As estudos tomados como base para a aplicação da acessibilidade do OA estão descritos abaixo.

Drigas et al. (2005) apontam quatro recomendações de acessibilidade ao apresentarem um sistema de gerenciamento de aprendizagem (LMS) para jovens surdos gregos: Informação bilíngue (texto e língua de sinais); alto nível de visualização; 
aprendizagem interativa e exploratória; e recursos para aprendizagem em grupos via videoconferência.

Khwaldeh, Matar e Hunaiti (2007) consideram quatro recomendações de acessibilidade a partir da criação de um sistema centralizado de e-learning para aula de matemática para crianças surdas na Jordânia: Fornecer informação de áudio de uma maneira visual, legendas para cada vídeo, figura e texto; fornecer um dicionário e glossário de termos; fornecer interfaces gráficas atrativas e efetivas; e fornecer níveis de dificuldades para avaliações.

Abreu, Prates e Bernardino (2010), apresentam em sua pesquisa três recomendações de acessibilidade para projetos de TICs para alfabetização de crianças surdas: Fornecer alternativas de textos equivalentes a conteúdo visual; oferecer alternativas equivalentes (legendas ou descrições textuais) para trechos de áudio em apresentações multimídia, dando preferência para vídeos em língua de sinais; e considerar a possibilidade de se propor situações onde seja possível realizar atividades em grupo ou em dupla.

Uma pesquisa de Lima (2014) levantou os principais elementos de acessibilidade para usuários surdos. De acordo com sua pesquisa, dos 13 itens apresentados ao sujeitos da pesquisa, sete foram considerados relevantes ou totalmente relevantes por mais de $60 \%$ dos respondentes. Em ordem crescente de relevância são: Presença da Língua de Sinais/Libras em ambientes digitais, Apresentação de conteúdos digitais em diferentes formatos (texto, imagem, vídeo e som) e em hipermídia, Vídeos em Libras com Legenda em português, Controle do usuário sobre as apresentações das informações (Voltar, adiantar, parar, começar), Apresentação do conteúdo em textos escritos em português disponíveis também por meio de vídeos dinâmicos em Libras, Disponibilizar dicionários digitais em Libras para consulta do usuário, Presença de legendas (closed caption) em português escrito para vídeos.

Canal (2015) em sua dissertação propõe nove recomendações de acessibilidade para surdos para avaliação baseada em computador: Vídeo em língua de sinais; apresentação alternativa para áudio; apresentação alternativa para texto escrito; apresentação alternativa para conteúdo visual; conteúdo; dicionários e glossários de termos; navegação; colaboração entre os estudantes; e feedback.

Tomando por base as pesquisas de Drigas et al. (2005), Khwaldeh, Matar e Hunaiti (2007), Abreu, Prates e Bernardino (2010), Lima (2014) e Canal (2015), o GT OBAMA - CARE definiu cinco recomendações para que um OA atinja os dois eixos que abordam os princípios de acessibilidade, segundo W3C-WAI: assegurar uma transformação harmoniosa e tornar o conteúdo compreensível e navegável. Foram elas: (1) Informação bilíngue (Libras e texto escrito); (2) Informação de áudio apresentado visualmente; (3) Níveis de dificuldades para avaliações; (4) Feedback escritos e sinalizados; (5) Dicionários e glossários digitais em Libras.

As recomendações 1, 2, 4 e 5 justificam-se por considerar a Língua de Sinais como primeira língua para o usuário surdo. As recomendações 2 e 4 por considerar que os sujeitos surdos necessitam em seus processos de aprendizagem um acompanhamento maior de acertos e erros e níveis gradativos de dificuldades de avaliações, como demonstram as pesquisas citadas. 
Vale ressaltar que, essas recomendações deverão ser validadas a posteriori junto com a validação da acessibilidade do OA ContaKg.

Pensando, então nas cinco recomendações iniciais e considerando que o software educativo ContaKg fornece o feedback, identificamos que seria possível contemplar os itens 1, 2 e 4 inserindo os vídeos em Libras.

Foi feita uma transcrição de todo áudio, texto e outros itens que necessitasse serem sinalizados em Libras. Ao todo, foram identificados 49 itens a serem sinalizados.

O passo seguinte era glosar os 49 itens para a sinalização em Libras. Optou-se pelo sistema de glosas devido a facilidade de leitura proporcionada, visto que são palavras de uma língua oral usadas para representar sinais manuais de sentido próximo e que são grafadas com letras maiúsculas.

As glosas foram validadas por um professor surdo. Com a glosa pronta, foi realizada a gravação em vídeo da sinalização em Libras. Posteriormente foram feitos testes que consistiam na apresentação desse vídeos a pessoas com deficiência auditiva, para saber se a tradução em Libras estava coerente com texto do modo que se esperava para a incorporação dela no OA.

Para a utilização no Unity, os vídeos foram editados e convertidos em GIFs (Graphics Interchange Format), um formato compactado, mais leve, que não traria ao OA uma lentidão no processamento.

Originalmente o Conta $\mathrm{Kg}$ foi feito na linguagem JavaScript juntamente com HTML (HyperText Markup Language) e CSS (Cascading Style Sheets). Todo o conteúdo precisou ser adequado ao Unity, e todas as interações foram refeitas usando a linguagem C\#, pré-definida na plataforma escolhida. Foram implementados os GIFs de modo que sempre que o cursor do mouse estiver em cima de um texto, a tradução em Libras fique visível.

\section{Resultados e Discussões}

Nesta seção serão discutidos os resultados e dificuldades encontrados no desenvolvimento do OA acessível.

Para a realização da incorporação da Libras no OA, foi criada uma glosa com todos os 49 itens, incluindo expressões, palavras e textos, de cada tela para ser gravada sua sinalização em Libras. Isto é, um documento contendo todas as informações textuais e suas respectivas adaptações para sinalização em Libras. Com isso, foi realizada gravação da sinalização e iniciada implementação da versão acessível do OA. O grupo contou com o apoio de uma equipe de multimídia que auxiliou no processo de gravação dos vídeos. No quadro 2 está exemplificada a tradução de um texto do OA para a glosa.

Quadro 2 - Exemplo de Glosas do OA ContaKg

\begin{tabular}{|l|l|}
\hline \multicolumn{1}{|c|}{ Texto português escrito } & \multicolumn{1}{|c|}{ Glosa para tradução em Libras } \\
\hline $\begin{array}{l}\text { Veja novamente o gráfico para ter certeza } \\
\text { da resposta correta. }\end{array}$ & $\begin{array}{l}\text { VEJA DE NOVO GRÁFICO TER } \\
\text { CERTEZA RESPOSTA CORRETA. }\end{array}$ \\
\hline
\end{tabular}

Fonte: Elaborado pelos autores. 
Após as gravações em Libras, os vídeos foram editados e transformados em GIFs. Devido a uma incompatibilidade desse formato com a plataforma Unity, foi necessário um procedimento de configuração, que consistiu em: Transformar o GIF em uma SpriteSheet ${ }^{3}$; importar o arquivo criado para o Unity e, a partir da imagem gerada, criar uma animação 2D para que ela funcione como um GIF, para enfim adicionar a animação na cena escolhida para sempre que o mouse passar por algum texto, palavra ou número seja visualizado o sinal em Libras correspondente.

Para o GT OBAMA - CARE esse foi o primeiro contato com o Unity, um motor

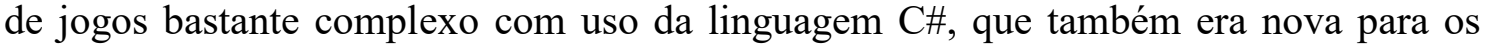
integrantes. Vale ressaltar os desafios de produzir conhecimento ao passo que se compreende como a plataforma funciona e realiza-se um projeto de qualidade, sabendo adequar a linha de pesquisa em algo que seja válido. Apesar disso, todas a dificuldades encontrada foram tomadas como desafios e isso construiu um processo de aprendizagem e superação, potencializando o conhecimento.

A adaptação do Conta $\mathrm{Kg}$ está feita, mas o OA ainda não está finalizado. As tabelas e gráficos ainda estão sendo desenvolvidos, pois o processo de aprendizagem da plataforma e a produção do OA estão acontecendo simultaneamente.

\section{Conclusões}

A tecnologia educacional assume um papel cada vez mais importante na perspectiva inclusiva como recurso de acesso a informações e conhecimentos a pessoas com deficiência. Para o indivíduo surdo, a acessibilidade comunicacional perpassa necessariamente pelo acesso às informações através de recursos visuais como Tradução e Interpretação de Libras, a Legenda para Surdos e Ensurdecidos (LSE), a Escrita de Sinais, entre outros.

Nesse sentido, o objetivo deste trabalho foi relatar a adaptação do Objeto de Aprendizagem Conta Kg para pessoas surdas, que foi concluído com o uso dos GIFs que traduziu todo o texto escrito para Libras, como forma de contribuir para o uso de tecnologias digitais por estudantes surdos, configurando-se em uma possibilidade de incluir esse público no contexto educativo e expandir o uso das tecnologias para o ensino da estatística.

A acessibilidade comunicacional incluída no OA, precisa agora passar por um estudo de recepção com a comunidade surda. E, para isso, será feito o uso do OA Conta $\mathrm{Kg}$, em uma escola pública, com estudantes surdos do Ensino Fundamental da Educação Básica. Um grupo de estudantes surdos vão vivenciar as duas modalidade do OA: sem acessibilidade e com acessibilidade e responderem a um questionário aplicado comparativo após cada vivência. Com o estudo de recepção poderemos avaliar o que tornará o OA mais acessível ao público surdo para que sejam feitas novas implementações de acessibilidade. Há, ainda, a intenção de realizarmos uma avaliação para identificar o nível de acessibilidade que será feita pelo GT Acessibilidade, tomando por base as Diretrizes de Acessibilidade para Conteúdo Web (WCAG) 2.0.

\section{Referências}

\footnotetext{
${ }^{3}$ Arquivos que contém sequências inteiras de imagens para compor uma animação. Utilizou-se o site ezgif.com.
} 
Bahia, S.; Trindade, J. P. (2010) O potencial das tecnologias educativas na promoção da inclusão: três exemplos. Educação, Formação \& Tecnologias, 3 (1), p. 96-110. [Online], disponível a partir de: $<$ http://eft.educom.pt $>$.

Brasil. (2017) Base Nacional Comum Curricular. Brasília: MEC. Disponível em: $<$ http://basenacionalcomum.mec.gov.br/images/BNCC_20dez_site.pdf $>$. Acesso em: 11 de maio de 2020.

Coutinho, Maria Dolores Martins da Cunha. (2015) A Constituição de Saberes num Contexto de Educação Bilíngue para Surdos em Aulas de Matemática numa Perspectiva de Letramento. Campinas, SP: [s.n] 2015.

Dresch, A; Lacerda, D. P.; Antunes Junior, J. A. V. (2015) Design Science Research: método de pesquisa para avanço da ciência e tecnologia. Porto Alegre: Bookman, 2015.

Flor, C. S.; Vanzin, T.; Ulbricht, V. R. (2013) Recomendações da WCAG 2.0 (2008) e a acessibilidade de surdos em conteúdos da web. Revista Brasileira de Educação Especial, v.19, p. 161-168, 2013.

Gagliardi, C. \& Barrella, F. F. (1986). Uso da informática na educação do deficiente auditivo: um modelo metodológico. Em Sociedade Brasileira de Psicologia (Org.), Anais da XVI Reunião Anual de Psicologia (pp. 120-123). Ribeirão Preto: SBP.

Giroto, C. R. M; Poker, R. B; Omote, S. (2012) Educação Especial, formação de professores e o uso das tecnologias de. Informação e comunicação: a construção de práticas pedagógicas inclusivas. In: Giroto, C. R. M; Poker, R. B; Omote, S. (Org.). (2012) As tecnologias nas práticas pedagógicas inclusivas. Marília: Oficina Universitária; São Paulo: Cultura Acadêmica, p. 11-24.

Góes, A. R. S. and Gomes, R. C. (2011) E-acessibilidade para surdos. Revista Brasileira de Tradução Visual. Recife, v.7, n.7.

Lewis, M.; Jacobson, J. (2002) Introdução. Comum. ACM 45, 1 (janeiro de 2002), $27-$ 31. DOI: < https: //doi.org/10.1145/502269.502288>.

Lima, F. R. (2014). Um estudo teórico e prático sobre acessibilidade da informação para surdos. TCC submetido ao curso de Gestão da Informação, da Universidade Federal de Pernambuco. Recife. 70 p.

Maia, et al. (2017) OBAMA: um Repositório de Objetos de Aprendizagem para Matemática. In: VI Congresso Brasileiro de Informática na Educação - CBIE 2017, Anais dos Workshops do VI Congresso Brasileiro de Informática na Educação WCBIE 2017, Recife: WCBIE 2017, p. 300-307.

Maia, et al. (2018) Software Educativo ContaKg. In: III Congresso sobre Tecnologias na Educação - Ctrl+E, UFCE, Anais, p. 590-596. Disponível em: <http://ceurws.org/Vol-1877/CtrlE2017_MC_10.pdf>. Acesso em: 11 de maio de 2020.

Melo, A. M. (2015) Acessibilidade em Objetos de Aprendizagem. In: Braga, Juliana (Org.). Objetos de Aprendizagem Volume 1: introdução e fundamentos. Santo André: UFABC, 2015, p. 93-111. Disponível em: $<$ pesquisa.ufabc.edu.br/intera/?page_id=370>. Acesso em: 11 de maio de 2020.

Myers, M. D.; Venable, J. R. (2014). A Set of Ethical Principles for Design Science Research in Information Systems. Information Management, vol. 51 no. 6, p. 801-809. 
Obilade, T. T. A. (2015) Comparative Study of the Cognitive Function of the HearingImpaired and Non-Hearing Impaired in Two Primary Schools in Lagos State, Nigeria. International Archives of Medicine, v.8, 2015.

Oliveira, et al. (2018) Objetos de Aprendizagem para Matemática: yes we can!. In: II Congresso sobre Tecnologias na Educação - Ctrl+E, UFPB, Anais, p. 744-750. Disponível em: $<$ http://ceur-ws.org/Vol-2185/CtrlE_2018_paper_122.pdf $>$. Acesso em: 11 de maio de 2020.

Pereira, M. C. da C. (2014) O ensino de português como segunda língua para surdos: princípios teóricos e metodológicos. Educar em Revista, Curitiba, Brasil, Edição Especial n. 2/2014, p. 143-157. Editora UFPR.

Rodrigues, D. (2012) As tecnologias de informação e comunicação em tempo de educação inclusiva In: Giroto, C. R. M; Poker, R. B; Omote, S. (Org.). (2012) As tecnologias nas práticas pedagógicas inclusivas. Marília: Oficina Universitária; São Paulo: Cultura Acadêmica, p.25-40.

Santarosa, L. M. C; Conforto, D. (2015) Tecnologias Móveis na inclusão escolar e Digital de estudantes com Transtornos de espectro autista. Rev. Bras. Ed. Esp., Marília, v. 21, n. 4, p. 349-366, Out.-Dez.

Surdez e perda auditiva. Organização Mundial da Saúde (OMS), 15 de março de 2019. Disponivel em: <OMS-https://www.who.int/news-room/fact-sheets/detail/deafnessand-hearing-los $>$. Acesso em: 11 de maio de 2020.

Venable, J. R. (2006) The role of Theory and Theorising in Design Science Research. DESRIST, v. 24-25, p. 1-18, 2006.

Viana, et al. (2019) Construindo Trajetórias Acessíveis para Pessoas Surdas na Plataforma OBAMA. In: Encontro Nacional de Acessibilidade Cultural - ENAC, 7, 2019, Porto Alegre (RS): UFRGS, 2019.

Viana, et al. (2018) Discutindo a acessibilidade e usabilidade dos objetos de aprendizagem na plataforma OBAMA. Anais do XXIV Workshop de Informática na Escola (WIE 2018).

Viana, F. R; Barreto, M. C. (2014) O ensino de matemática para alunos com surdez: Desafios docentes, Aprendizagens discentes. Curitiba, PR: Editora CRV.

Xavier, L. M. F. da S. (2011) O uso das TIC em salas de aula inclusivas: Atitudes e práticas de professores do $1^{\circ}$ ciclo. Dissertação (Mestrado em Ciências da Educação). Escola Superior de Educação de Lisboa. 292f. 